\title{
INEQUALITIES FOR THE INCENTER SIMPLICES
}

\author{
MA TONGY, ZHAO LINGZHI AND YUAN JUN
}

Abstract. Let $I_{i}(i=0,1,2, \ldots, n)$ denote the incenter of facet $F_{i}$ of an $n$-dimensional simplex $\Omega_{A}$ and we call $\Omega_{I}=\operatorname{conv}\left\{I_{0}, I_{1}, \ldots, I_{n}\right\}$ the incenter simplex of $\Omega_{A}$. In [3], L. H. Tang and G. S. Leng conjectured

$$
V\left(\Omega_{I}\right) \leqslant \frac{1}{n^{n}} V\left(\Omega_{A}\right),
$$

with equality if and only if $\Omega_{A}$ is a regular simplex. In this paper, we give a positive answer of the conjecture. Further, we improve the condition of the equality holds.

Mathematics subject classification (2000): 52A40, 52A20.

Key words and phrases: tangent points simplex, orthocentric simplex, incenter simplex.

\section{REFERENCES}

[1] Q. J. MaO, Q. R. ZUO, A geometric inequality of tangency-point simplex, Mathematics in Practics and Theory, 17, (4) (1987), 71-75.

[2] H. M. Su, An inequality for a simplex, Bulletin of Maths, 5, (1985), 43-46.

[3] L. H. TANG, G. S. LENG, Two new inequalities for tetrahedron, Maths Competition, Changsha, Education Press of Hunan, (1994), 88-93.

[4] L. YANG, J.ZH. ZHANG, The notation of rank in abstract distance space, Journal of University of Science and Technology of China, 10, (4) (1980), 52-65.

[5] L. YANG, J. ZH. ZHANG, The geometric proof of a algebra theorem, Journal of University of Science and Technology of China, 11, (4) (1981), 45-49.

[6] L. YANG, J. ZH. ZHANG, A class of geometric inequalities on finite points(Chinese), Acta Mat. Sinica, 23, (5) (1980), 740-749.

[7] Y.ZHANG, A conjecture of pedal simplex, Journal of Systems Sci and Math Sci, 12, (4) (1992), 371-375. 